\title{
Authors' reply to comment by Tailor et. al on "Academic cosmetic gynecology and energy-based therapies: ambiguities, explorations, and FDA advisories"
}

\author{
Bobby Allen Garcia ${ }^{1}$ (D) Jack Pardo ${ }^{2}$ \\ Received: 15 April 2019 / Accepted: 3 May 2019 / Published online: 4 June 2019 \\ (C) The International Urogynecological Association 2019
}

Dear Editors,

We thank Visha Tailor and colleagues for their interest in our recent publication on cosmetic gynecology and energybased therapies [1]. Although we seemingly adopt a different approach, I am confident that we share the same endpoints: taking better care of patients and decreasing adverse events.

Our two main intents in publication were to raise awareness about cosmetic gynecology and energy-based therapy. Let us begin with vaginal rejuvenation, the elephant in the room. As leaders of the International Urogynecological Association (IUGA) Special Interest Group in Cosmetic Gynecology, we understand just how severely a lack of standard definitions and objective cosmetic and functional outcomes has impaired development in this field. To address this, we have submitted a proposal to the IUGA Terminology and Standardization Committee that was accepted to begin a document on cosmetic gynecology. Then, once common definitions have been developed, a new, stronger foundation can be built, upon which comparative endpoints (safety and efficacy data) can be produced. "Academic cosmetic gynecology" is not so much a new term as it is our pledge to help in the development of these objective measures.

In the interim, however, we cannot ignore this topic simply because there is no defined nomenclature. In 2016, Americans spent US $\$ 15$ billion on cosmetic procedures, with 10,774 labiaplasties performed (a 23\% increase since 2015) [2]. An ongoing systematic review (by author BA Garcia) suggests

This reply refers to the letter available at https://doi.org/10.1007/s00192019-03959-0

Bobby Allen Garcia

garcia.bobby@gmail.com

1 Louisiana State University Health Sciences Center, New Orleans, LA, USA

2 Urogyn \& Cosmetic Gyn, Clinica Ginestetica, Santiago, Chile that $69 \%$ of these procedures are performed by plastic surgeons, with only $25 \%$ by gynecologists.

We absolutely agree that vigilance must be observed in the characterization and treatment of women with genitourinary syndrome of menopause. We further concur that a hypoestrogenic state is best treated with localized replacement of hormones and that it can be offered as an option for women with a history of breast cancer. The theoretical mechanism posited for the improvement of sexual function in women undergoing energy-based therapy, while intuitive, is admittedly in our editorial, conjecture. Most importantly, we agree that more objective, randomized, placebo-controlled research needs to be carried out to properly vet this technology.

Much like cosmetic gynecology, there is a breadth of information available that can be confusing, not only to patients but also to providers. Energy-based therapies are another therapeutic modality, an arrow to be added to our quiver of treatment options for urogynecological conditions. As we continue to develop research protocols and produce data, we will be able to take better aim and refine our treatment targets.

\section{References}

1. Garcia B, Pardo J. Academic cosmetic gynecology and energy-based therapies: ambiguities, explorations, and FDA advisories. Int Urogynecol J. 2019;30(1):1-2

2. 2016 Cosmetic Surgery National Data Bank Statistics. https://www. surgery.org/sites/default/files/ASAPS-Stats2016.pdf Date Accessed $4 / 11 / 19$.

Publisher's note Springer Nature remains neutral with regard to jurisdictional claims in published maps and institutional affiliations. 